\title{
Investigation the Effects of Neutron Source Number and Arrangement in Landmines Detection by Thermal Neutron Capture Gamma Ray Analysis
}

\author{
Leila Abdolahi Shiramin, Seyed Farhad Masoudi * \\ Physics Department, K.N. Toosi University of Technology, P.O. Box 15875-4416, Tehran, Iran \\ Email: *masoudi@kntu.ac.ir
}

Received September 17, 2011; revised December 8, 2011; accepted December 28, 2011

\begin{abstract}
Thermal neutron capture can be used as a successful technique for detection of non-metallic landmines via the detection of their constituent like nitrogen. Recently, it has been shown that the detection of $10.829 \mathrm{MeV}$ photons from the ${ }^{14} \mathrm{~N}(\mathrm{n}, \gamma){ }^{15} \mathrm{~N}$ reaction can be used for finding the landmines. In this method a high-energy neutron source like ${ }^{241} \mathrm{Am}-\mathrm{Be}$ inside water as a moderator is used to have thermal neutron. In this paper we have investigated the effects of the number of neutron sources and their orientation on the gamma ray spectrum by using MCNP4C code. The best case for number of sources and their positions and orientations have been achieved corresponding to maximum flux of $10.829 \mathrm{MeV}$ photons.
\end{abstract}

Keywords: Monte Carlo Simulation; Landmine Detection; Thermal Neutron Capture; Neutron Source; Source Arrangement

\section{Introduction}

During the recent years many efforts have been made to improve nuclear methods for the detection of landmines [1-8]. The most important advantage of methods based on neutron reactions is that neutrons have high penetration ability and the detection of landmines is possible even when they are hidden at the high depth. Nuclear techniques are based on that the explosives contain hydrogen, carbon, nitrogen and oxygen in variable concentration. For example, elementary compositions of some common explosives are: TNT- $\mathrm{C}_{7} \mathrm{H}_{5} \mathrm{~N}_{3} \mathrm{O}_{6}, \mathrm{RDX}-\mathrm{C}_{3} \mathrm{H}_{6} \mathrm{~N}_{6} \mathrm{O}_{6}$, Hexogen- $\mathrm{C}_{4} \mathrm{H}_{8} \mathrm{~N}_{8} \mathrm{O}_{8}$ [9]. So the problem of explosives identification reduces to the problem of identification of light elements.

One method of landmines detection is based on measurement of the $10.829 \mathrm{MeV}$ gamma-ray from the ${ }^{14} \mathrm{~N}(\mathrm{n}, \gamma){ }^{15} \mathrm{~N}$ reaction [10]. This procedure is advantageous because the gamma-rays of about $11 \mathrm{MeV}$ do not occur in natural background. The only exception is ${ }^{29} \mathrm{Si}$ which emits gamma-ray of $10.607 \mathrm{MeV}$ via interaction with thermal neutrons, but with low percentage. The abundance of ${ }^{29} \mathrm{Si}$ in soil is very low in comparison with abundance of ${ }^{14} \mathrm{~N}$ in landmine.

In present work, MCNP4C code is applied for simulation of configuration of a hidden landmine in soil and

\footnotetext{
${ }^{*}$ Corresponding author.
}

investigation of the source number and their placement effects on gamma-ray spectrum. The maximum value for $10.829 \mathrm{MeV}$ photon fluxes that depends on the numbers and orientations of the sources has been achieved by using the result of MCNP4C simulation.

\section{Material and Methods}

In this paper, the MCNP4C code for Monte Carlo simulation of particle transport was employed to obtain the best configuration corresponding to best values of 10.829 $\mathrm{MeV}$ neutron flux. An Am-Be neutron source as an isotropic source has been used with neutron spectrum shown in Figure 1 [11]. The neutron source which is 12 $\mathrm{cm}$ in length and $3 \mathrm{~cm}$ in diameter was located horizontally in the box of water with dimensions of $60 \times 60 \times 24$ $\mathrm{cm}^{3}$. Distance of the box of water and the surface of soil is $1 \mathrm{~cm}$. A landmine (TNT) in cylindrical geometry, $5 \mathrm{~cm}$ in radius and $5 \mathrm{~cm}$ in height, was embedded at $5 \mathrm{~cm}$ depth from the surface of soil with dimensions of $60 \times 60$ $\times 11 \mathrm{~cm}^{3}$. NaI(Tl) $3 \times 3$ detector was positioned at the center of water box to take the spectrum of photons (Figure 2) [12]. $\mathrm{NaI}(\mathrm{Tl})$ scintillator as $\gamma$-ray detector has been suggested by considering its characteristics such as the low cost of the crystal, the good light output and a low internal background in the energy region around 11 $\mathrm{MeV}$ due to thermal neutron capture processes on the nuclei of the crystal [13]. 


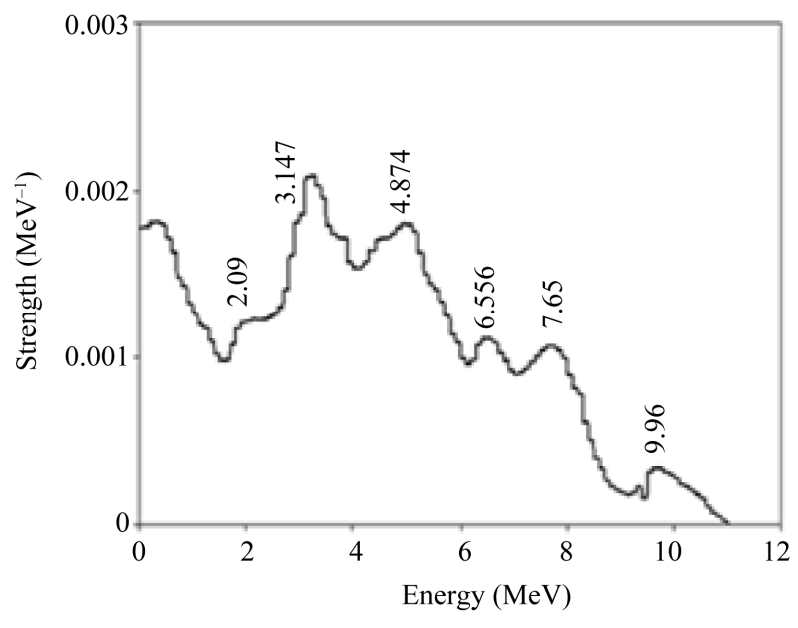

Figure 1. Neutron energy spectrum of Am-Be source.

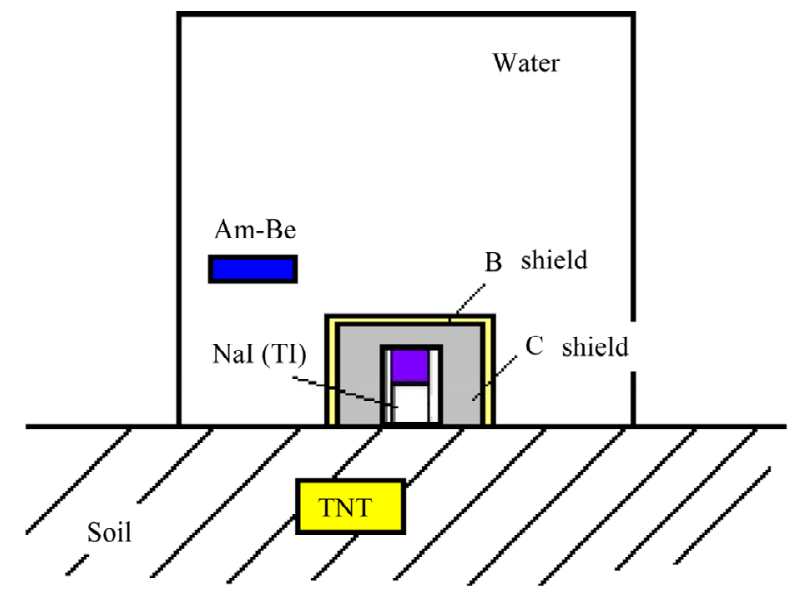

Figure 2. A schematic view of the configuration used in MCNP calculation.

In order to prevent the reaching of thermal neutrons to the detector, it was shielded by ${ }^{12} \mathrm{C}$ as a reflector of thermal neutrons [14] and was surrounded with ${ }^{10} \mathrm{~B}$ to absorb thermalized neutrons [15]. The diameter and height of the carbon shield are $18 \mathrm{~cm}$ and $12.09 \mathrm{~cm}$, respectively and that of boron shield are $19 \mathrm{~cm}$ and $13.09 \mathrm{~cm}$, respectively.

In geometry of two sources, they were located on the both sides of the detector and in 3 sources, one of sources was positioned on one side and two other sources were located on the other side. Geometry of four sources can be explained as two of sources were placed on one side and two other sources on the other side of detector with different directions. Geometry of four sources in the presence of symmetry is the same with single source, but four sources were located in four corner of water tank.

We carried out the calculation with different configurations of sources to investigate effect of placement of sources and determine the best configuration for maximum photon flux.

\section{Results and Discussions}

Figure 3 shows the gamma-ray spectrum of neutron capture by soil and landmine using F5 tally in MCNP code for the best set up.

In order to investigate the effect of placement of sources in the water tank on photon flux spectrum, the sources was placed at different configurations (different rotations about $z$-axis and different distances from the bottom of tank). Table1 shows some of the results of our simulations where $\mathrm{z}$ is the distance of source from the bottom of the water tank and $\alpha$ is the angle between the source axis and water tank axis. The MCNP calculations show that the number and placement of sources have effect on photon flux strongly. As shown in Table 1, the best set up is related to 3 sources geometry where one of the sources is on one side of the detector with a $45^{\circ}$ rotation about the $z$-axis at a distance of $2 \mathrm{~cm}$ from the bottom of the water tank and two of them are on the other hand horizontally and vertically at a distance of $3 \mathrm{~cm}$ from the bottom of the water tank. Also Table 1 shows that the symmetry in the arrangement of the sources doesn't have positive effect on increment of photon flux.

Figure 4 shows background-subtracted spectrum for the best set up of 1, 2, 3 and 4 sources. In the background spectrum a trace of $10.829 \mathrm{MeV}$ peak is seen, which is smaller than $10.829 \mathrm{MeV}$ peak in the spectrum of photon in the presence of TNT. This is due to neutron capture in $\mathrm{N}$ in air under the water tank and surrounding. As shown in Figure 4 photon flux in presence of 4 sources is the same with flux of 3 sources because with increment of number of the source, the volume of water as a neutron moderator decreases and system can't produce more thermal neutrons and so neutron capture events and photon flux decrease.

Simulations showed background in presence of 3

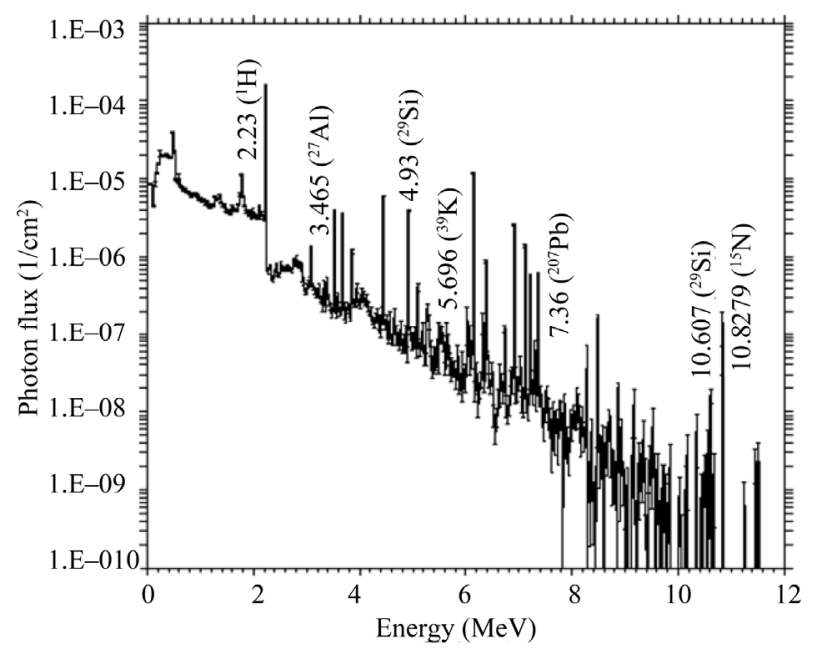

Figure 3. The gamma-ray spectrum of soil containing TNT in the presence of 3 sources. 


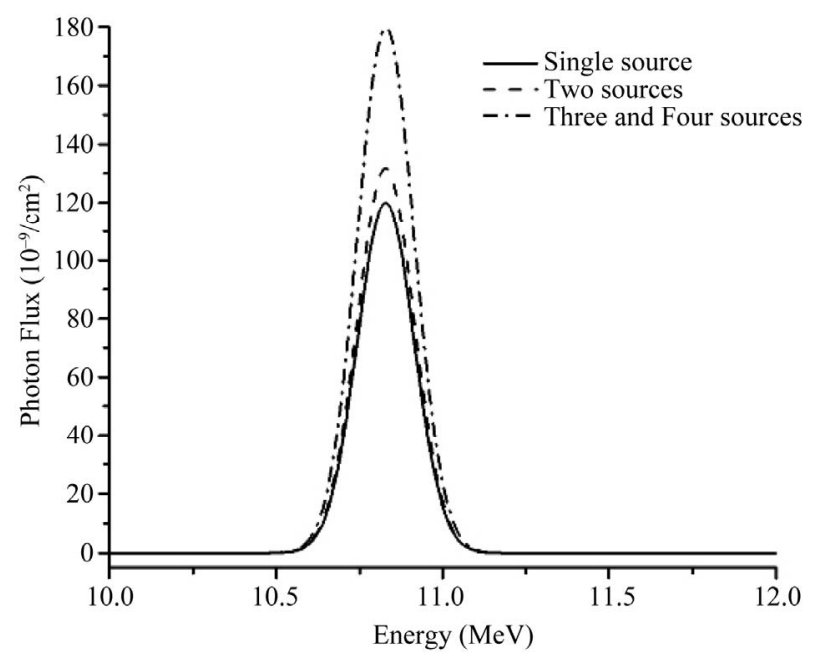

Figure 4. The photon flux for 1, 2, 3 and 4 sources corresponding to the best configurations (without background).

Table 1. The 10.829 MeV gamma-ray flux for different configurations of sources (TNT in center of soil).

\begin{tabular}{ccc}
\hline $\begin{array}{c}\text { Background at } \\
10.829 \mathrm{MeV}\left(\times 10^{-9}\right)\end{array}$ & Source number & $\begin{array}{c}\text { Flux of } \\
10.829 \mathrm{MeV}\left(\times 10^{-9}\right)\end{array}$ \\
\hline 2.14 & 1 & 54.68 \\
5.4 & 2 & 66.9 \\
& 3 & 117 \\
& $3(\alpha: 30)$ & 91 \\
8.32 & $3(\alpha: 60)$ & 25.1 \\
& $3(\mathrm{z}=3 \mathrm{~cm})$ & 49.68 \\
& $3(\mathrm{z}=2.5 \mathrm{~cm})$ & 56.3 \\
10.54 & 4 & 115 \\
3.25 & $4($ symmetrical $)$ & 71 \\
\hline
\end{tabular}

sources is low in comparison with 4 sources and its useful for obtaining of exact counts by $\mathrm{NaI}(\mathrm{Tl})$.

\section{Conclusions}

The MCNP calculations showed that the arrangement of the sources and the number of them have important influence on landmines detection by thermal neutron capture technique. In this paper the effect of the presence and arrangement of 2, 3 and 4 sources was studied on the capture events and $10.829 \mathrm{MeV}$ photon flux.

Results show that the maximum photon flux is corresponding to 3 sources geometry where one of the sources is on one side of the detector with a $45^{\circ}$ rotation about the $z$-axis at a distance of $2 \mathrm{~cm}$ from the bottom of the water tank and two of them are on the other hand horizontally and vertically at a distance of $3 \mathrm{~cm}$ from the bottom of the water tank Background counts in this geometry are low in comparison with 4 sources and this is advantageous for obtaining exact counts and biological effects.

\section{REFERENCES}

[1] J. C. Campbell and A. M. Jacobs, "Detection of Buried Landmines by Backscatter Imaging," Nuclear Science and Engineering, Vol. 110, 1992, pp. 417-424.

[2] M. Maucec and R. J. de Meijer, "Monte Carlo Simulations as a Feasibility Tool for Non-Metallic Landmine Detection by Thermal-Neutron Backscattering," Applied Radiation and Isotopes, Vol. 56, No. 6, 2002, pp. 837846. doi:10.1016/S0969-8043(02)00064-7

[3] K. M. Dawson-Howe and T. G. Williams, "The Detection of Buried Landmines Using Probing Robots," Robotics and Autonomous System, Vol. 23, No. 4, 1998, pp. 235243. doi:10.1016/S0921-8890(98)00010-4

[4] A. V. Kuznetsov, A. V. Evsenin and I. Y. Gorshkov, "Detection of Buried Explosives Using Portable Neutron Sources with Nanosecond Timing," Applied Radiation and Isotopes, Vol. 61, No. 1, 2004, pp. 51-57. doi:10.1016/j.apradiso.2004.02.016

[5] M. Lunardon, G. Nebbia, S. Pesente, G. Viesti and V. Filippini, "Detection of Landmines by Using $14 \mathrm{MeV}$ Neutron Tagged Beams," Applied Radiation and Isotopes, Vol. 61, No. 1, 2004, pp. 43-49. doi:10.1016/j.apradiso.2004.02.015

[6] M. Maucec and C. Rigollet, "Monte Carlo Simulations to Advance Characterisation of Landmines by Pulsed Fast/ Thermal Neutron Analysis," Applied Radiation and Isotopes, Vol. 61, No. 1, 2004, pp. 35-42. doi:10.1016/j.apradiso.2004.02.014

[7] A. Pazirandeh, M. Azizi and S. F. Masoudi, "Monte Carlo Assessment of Soil Moisture Effect on High-Energy Thermal Neutron Capture Gamma-Ray by ${ }^{14} \mathrm{~N}$," Applied Radiation and Isotopes, Vol. 64, No. 1, 2006, pp. 1-6. doi:10.1016/j.apradiso.2005.05.053

[8] D. R. Ochbelagh, H. M. Hakimabad and R. I. Najafabadi, "The Soil Moisture and Its Effect on the Detection of Buried Hydrogenous Material by Neutron Backscattering Technique," Radiation Physics and Chemistry, Vol. 78, No. 5, 2009, pp. 303-306. doi:10.1016/j.radphyschem.2009.02.002

[9] J. Obhodas, D. Sudac, K. Nad, V. Valkovic, G. Nebbia and G. Viesti, "The Soil Moisture and Its Relevance to the Landmine Detection by Neutron Backscattering Technique," Nuclear Instruments and Methods in Physics Research Section B, Vol. 213, 2004, pp. 445-451. doi:10.1016/S0168-583X(03)01587-8

[10] S. Pesente, M. Cinausero, D. Fabris and E. Fioretto, "Effects of Soil Moisture on the Detection of Buried Explosives by Radiative Neutron Capture," Nuclear Instruments and Methods in Physics Research Section A, Vol. 459, No. 3, 2001, pp. 577-580. doi:10.1016/S0168-9002(00)01058-5

[11] H. M. Hakimabad, A. V. Noghreiyan and H. Panjeh, "Improving the Moderator Geometry of an Anti-Personnel Landmine Detection System," Applied Radiation and Isotopes, Vol. 66, No. 5, 2008, pp. 606-611.

[12] H. T. Anbaran, R. I. Najafabadi and H. M. Hakimabad, "Optimization of a Detector Collimator for Use in a Gamma-Ray Backscattering Device for Anti-Personal 
Landmines Detection," Applied Sciences, Vol. 9, No. 9, 2009, pp. 2168-2173.

[13] E. Chukhaev and A. I. Melnikov, "CsI(T1) Scintillators as $\gamma$-Ray Detectors for the Identification of Hidden Explosives," Nuclear Instruments and Methods in Physics Research Section A, Vol. 471, No. 1-2, 2001, pp. 234-238. doi:10.1016/S0168-9002(01)00979-2

[14] C. P. Datema, V. R. Bom and C.W. E. van Eijk, "Experimental Results and Monte Carlo Simulations of a Land- mine Localization Device Using the Neutron Backscattering Method," Nuclear Instruments and Methods in Physics Research Section A, Vol. 48, No. 1-2, 2002, pp. 441-450. doi:10.1016/S0168-9002(02)00402-3

[15] H. Akkurt, J. Wagner and K. Eckerman, "Hand Held Instruments for Landmine Detection: View from Radiation Dosimetry," Nuclear Instruments and Methods in Physics Research Section A, Vol. 579, No. 1, 2007, pp. 391-394. doi:10.1016/j.nima.2007.04.097 